\title{
Correspondence
}

Editor: Ian Pullen

Contents: The essential psychotherapies/Treatment resistance in schizophrenia/Lithium neurotoxicity at normal therapeutic levels/Court diversion/ Integrated psychological therapy for schizophrenia/ Epidemiology of vascular dementia/Offspring of parents with drinking problems/Münchhausen's syndrome?/Community Treatment Orders/Mania and Down's syndrome/Low serum cholesterol and serotonin receptor subtypes/Concepts of illness and disease/Neurological complications of anorexia nervosa.

\section{The essential psychotherapies}

SIR: Andrews' annotation concerning psychodynamic psychotherapy (Journal, April 1993, 162, 447451) comes unequivocally to the conclusion that while cognitive therapy is better than placebo, the current evidence is that this is not true for psychodynamic psychotherapy. He believes psychodynamic psychotherapy is not cost-effective and may also be harmful.

Andrews' argument about the efficacy of psychodynamic therapy relies mainly on a discussion of recent meta-analytic studies, but, in reporting them, he downplays the complexity of the findings of two of these studies. Chrits-Christoph (1992) is more cautious than how Andrews represents him, citing a number of crucial limitations to the generalisability of his findings. Furthermore, Chrits-Christoph's paper does not contain any conclusions with respect to a placebo condition as Andrews implies. Rather, it contains references to "alternative non-psychiatric treatments", the descriptions of these alternative treatments turning out to be rather similar to supportive therapy. Additionally, Swartberg \& Stiles (1991) did find short-term psychotherapy (STPP) inferior to cognitive therapy in depression, but equally they did not find an advantage to cognitive therapy over STPP in general neurotic disorders.

There are also continuing methodological worries about comparative trials which should rightly lead to increasing scepticism over the value of meta-analytic studies. Kasdin (1986) argues that the pervasive methodological limitations of the studies which are fed into these meta-analyses must limit their value. One particular problem lies in the use of outcome measures which are more suited to measuring change in one aspect of a comparative trial, and there is known to be a bias in most comparative trials in favour of the theoretical orientation of the first main author. Because cognitive therapists have been more diligent researchers than psychodynamic ones, more studies have been done by them and meta-analyses consequently may show bias in this direction.

Further, Andrews misrepresents two important papers which do deal with research into psychodynamic psychotherapy. An important meta-analytic paper (Howard et al, 1986) gives support to the likely efficacy of psychodynamic therapy by showing the existence of a dose-effect relationship. However, in Andrews' article the paper is only cited in relation to another issue, without mention of its main finding. Andrews is unduly critical of one of the few recent papers on longer-term dynamic psychotherapy by Stevenson \& Meares (1992). He gives the impression that their impressive results with borderline patients are most unlikely to be due to the specific effects of the therapy as opposed to spontaneous remission, placebo response, or a ceiling effect. However, Stevenson \& Meares critically discuss these possibilities in their paper and give good, if circumstantial, evidence why they are unlikely.

Next Andrews dismisses single-case studies on the grounds that single successes fall within the confidence limits for error, but he mistakes the point of single-case studies which are clearly not important in an overall statistical argument. Rather, their value lies in bringing together the details of a treatment into a coherent narrative which reveals the mechanism of a significant process of change.

The discussion of the efficiency of psychodynamic psychotherapy is also unduly gloomy. Andrews' resource calculation is deeply flawed. It takes no account of rational assessment and allocation processes. Indeed, within the context of a rational decision-making strategy about therapy, it has been shown that significantly fewer patients are found to 
need long-term therapy, and offering a general service with a range of therapies can be cost-effective for a Health Maintenance Organisation (Bennett \& Wisneski, 1979).

Andrews' argument seems hostile and hasty. It continues the division between psychodynamic psychotherapy and cognitive therapy and that between psychodynamic psychotherapy and research. Both divisions have been profoundly unfruitful for the growth of knowledge and mutual respect. For this reason, among others, his argument is regrettable.

BENNETT, M. J. \& WISNESKI, M. J. (1979) Continuous psychotherapy within an HMO. American Journal of Psychiatry, 136, 1283-1287.

Chrits-Christoph, P. (1992) The efficacy of brief dynamic psychotherapy: a meta-analysis. American Journal of Psychiatry. 149, 151-158.

Howard, K. I., Kopta, S. M., Krause, M. S., et al, (1986) The doseeffect relationship in psychotherapy. American Psychologist, 41, 159-164.

KASDIN, A. E. (1986) Comparative outcome studies of psychotherapy: methodological issues and strategies. Journal of Consulting and Clinical Psychology, 54, 95-105.

Stevenson, J. \& Meares, R. (1992) An outcome study of psychotherapy for patients with borderline personality disorder. American Journal of Psychiatry, 149, 358-362.

SWAR TBERG, M. \& Stiles, T. C. (1991) Comparative effects of shortterm psychodynamic psychotherapy: a meta-analysis. Journal of Consulting and Clinical Psychology, 59, 704-714.

The Cassel Hospital

Chess Denman

Richmond

London TWI0

SIR: Andrews' call (Journal, April 1993, 162, 447-451) for greater valuation and teaching of 'good clinical care' is as encouraging as his implicit dismissal of dynamic psychotherapy as 'inessential' is tendentious. Dynamic psychotherapy is essential to good clinical care as he depicts it, requiring an appreciation of the principles of overdetermination, defensemechanisms, and of the inevitability of transference and countertransference within the therapeutic relationship. I have described a similar approach as supportive analytic therapy (Holmes, 1992).

Andrews makes a valid point in his call for controlled studies of analytic psychotherapy, but surely overstates his case in his efforts to discredit the dynamic approach in comparison with cognitive therapy. Effect sizes may generally be greater with cognitive-behavioural approaches, but nonetheless his own study (Andrews \& Harvey, 1981) showed an impressive effect size of $\mathbf{0 . 7 4}$ for dynamic therapies. He fails to discuss the limitations of meta-analysis, or to mention Horowitz et als attempts to tease out the differential indications of dynamic and behavioural approaches, showing, for example, how in the psychotherapy of abnormal grief, inhibited patients did better with an expressive approach while less mature patients benefited from a behavioural one (Horowitz et al, 1984).

Andrews' arithmetic purports to show that, by using dynamic psychotherapy, $15 \%$ of psychiatrists are only able to treat $0.5 \%$ of the patients, and raises an important point about manpower, but this again reveals his bias. Many of the patients treated in psychotherapy departments suffer from severe personality disorders, and, despite their statistical infrequency, often consume huge amounts of ineffective, non-psychodynamic time and resources. Rosser et al (1987) showed that long-term, in-patient dynamic therapy with such patients can be effective and cost-effective, if 'offset costs' such as medical salaries, drug bills, and welfare payments are included in the equation. Andrews' concern with the 'safety' of psychotherapy, and his awareness of the dangers of therapist abuse are laudable, but his insinuation that dynamic therapy is less safe than other forms of psychotherapy is based on no firm evidence, and can only be regarded as a slur that fits the overall polemic of his piece.

We are entering an era of creative collaboration between the psychotherapies. Cognitive therapists are beginning to work with transference and countertransference, and dynamic therapists are increasingly abandoning dogma for observation while rating, evaluating, and researching their methods. Ryle's (1991) 'cognitive analytic therapy' is an example of a time-limited dynamic therapy, appropriate for 'thirdparty funding', that illustrates this new spirit of cooperation. If Andrews' aim was to provoke debate it is to be welcomed, but if to revive old antagonisms and destructiveness, he does a disservice to our discipline.

ANDRews, G. \& HARvey, R. (1981) Does psychotherapy benefit neurotic patients? A reanalysis of the Smith, Glass and Miller data. Archives of General Psychiatry, 38, 1203-1208.

Holmes, J. (1992) Between Art and Science: Essays in Psychotherapy and Psychiatry. London: Routledge.

Horowitz, M., Marmar, C., Weiss, D., et al (1984) Brief therapy of bereavement reactions: the relationship of process to outcome. Archives of General Psychiatry, 41, 438-448.

Rosser, R., BirCh, S., Bond, H., el al (1987) Five-year follow-up of patients treated with in-patient psychotherapy at the Cassel Hospital for Nervous Diseases. Journal of the Royal Society of Medicine, 80, 549-555.

RyLE, A. (1991) Cognitive Analytic Therapy: Active Participation in Change. Chichester: Wiley.

North Devon District Hospital

JEREMY HOLMES

Barnstaple

Devon 